\title{
On the dynamic viscous permeability tensor symmetry
}

\author{
Camille Perrot, Fabien Chevillotte, and Raymond Panneton \\ Groupe d'Acoustique de l'Universite de Sherbrooke (GAUS), Department of Mechanical Engineering, \\ Universite de Sherbrooke, Quebec J1K 2R1, Canada \\ camille.perrot@usherbrooke.ca,fabien.chevillotte@usherbrooke.ca. \\ raymond.panneton@usherbrooke.ca \\ Jean-François Allard and Denis Lafarge \\ Laboratoire d'Acoustique de l'Université du Maine (LAUM), UMR CNRS 6613, Avenue Olivier Messiaen, \\ 72085 Le Mans Cedex 9, France \\ jean-francois.allard@univ-lemans.fr,denis.lafarge@univ-lemans.fr
}

\begin{abstract}
Based on a direct generalization of a proof given by Torquato for symmetry property in static regime, this express letter clarifies the reasons why the dynamic permeability tensor is symmetric for spatially periodic structures having symmetrical axes which do not coincide with orthogonal pairs being perpendicular to the axis of three-, four-, and sixfold symmetry. This somewhat nonintuitive property is illustrated by providing detailed numerical examples for a hexagonal lattice of solid cylinders in the asymptotic and frequency dependent regimes. It may be practically useful for numerical implementation validation and/or convergence assessment.

(C) 2008 Acoustical Society of America

PACS numbers: 43.50.Gf, 43.55.Ev, 43.20.Wd, 43.58.Ta [MS]

Date Received: March 18, 2008 Date Accepted: July 8, 2008
\end{abstract}

\section{Introduction}

The viscous permeability tensor plays, for long-wavelength sound propagation in fluidsaturated rigid-framed porous structures, a role similar to the electric permittivity tensor in conducting media. In a comprehensive demonstration of Adler" in his classical textbook "Porous Media: Geometry and Transports," while examining the properties of the permeability tensor for flow in spatially periodic structures, it is clearly established that, essentially as a consequence of the linearity of the Stokes equations system for spatially periodic structures, the permeability is a positive definite and symmetric tensor, and can thus be diagonalized on an orthonormal basis. Wang ${ }^{2}$ extends this property by showing that if the permeability is the same in two particular orthogonal transverse directions, the material is isotropic in all orthogonal transverse directions. There is an analog property in optics for the dielectric tensor of optically uniaxial cristals. ${ }^{3}$

In this express letter, emphasis is put on the-dynamic-permeability tensor (frequency dependent) for a hexagonal lattice of solid cylinders. Contrary to a simple cubic lattice, it is not intuitively clear this time that the orthogonal directions necessarily coincide with the principal permeability axes since these directions are geometrically distinguishable from one another. The demonstration is based on a direct extension in harmonic regime of a proof given by Torquato for symmetry property in static regime. ${ }^{4}$ Consequently, the purpose of this express letter is first to clarify mathematically the reasons why the dynamic permeability tensor is symmetric for spatially periodic structures having symmetrical axes which do not coincide with orthogonal pairs being perpendicular to the axis of three- and sixfold symmetry. Second, it is proposed to illustrate this somewhat nonintuitive property by providing numerical examples for a hexagonal lattice of solid cylinders in the asymptotic and frequency dependent regimes. Third, it is finally mentioned that a systematic check of the diagonal and nondiagonal terms of the dynamic permeability tensor provides a fast and useful argument for convergence assessment of a numerical computation on this type of local periodic geometry. 
The flow of a viscothermal fluid in a motionless homogeneous porous structure was described by D.L. based on ideas borrowed from electrodynamics as follows. ${ }^{5}$ The angular frequency is $\omega$ and the time dependence is $\exp (-i \omega t)$. The statistical properties of the porous frame can be defined in homogenization volumes with dimensions much smaller than the wavelength of the acoustic waves that propagate in the saturating fluid. The microscopic quantities that describe the flow (pressure $p$, velocity v) present variations at the microscopic scale in the homogenization volume. To smooth out these variations and leave only the macroscopic variations, angular brackets \langle\rangle , indicating a fluid-phase average, are introduced. By definition, it relates the macroscopic variable $\langle a\rangle$ to the microscopic variable $a$ by

$$
\langle a\rangle(\mathbf{r}, t)=\int I(\mathbf{r}+\mathbf{x}) f(\mathbf{x}) a(\mathbf{r}+\mathbf{x}, t) d^{3} \mathbf{x},
$$

where $I$ is the characteristic function of the fluid phase ( 1 in the fluid $\Omega_{f}$ and 0 in the solid $\Omega_{s}$ ), and $f$ is a filtering smooth function nonzero only in some (homogenization) region surrounding $\mathbf{x}=\mathbf{0}$ and normalized to unity $\int I(\mathbf{r}+\mathbf{x}) f(\mathbf{x}) d^{3} \mathbf{x}=1 . f(\mathbf{x})$ is typically a hat-shaped curve which is formed by a central plateau and two decreasing regions. The length $L$ of the plateau and decreasing regions is chosen large compared to the material's local characteristic size $(L>a)$, so that the averaging operation performed through Eq. (1) has the effect of decreasing the small microscopic fluctuations due to structure inhomogeneities. The so smoothed fields are thus varying at a much larger scale (wavelength $\lambda \gg L$ ) than the material's local characteristic size. The discontinuity of $I$ at the contact surface $\partial \Omega$ between solid and fluid leads to the spatial average theorem

$$
\langle\boldsymbol{\nabla} a\rangle(\mathbf{r}, t)=\boldsymbol{\nabla}\langle a\rangle(\mathbf{r}, t)+\int_{\mathbf{x} \in \partial \Omega} f(\mathbf{x}-\mathbf{r}) \mathbf{n}(\mathbf{x}) a(\mathbf{x}, t) d S .
$$

The vector $\mathbf{n}$ is the unit outward normal vector to the fluid domain. When the wavelengths become comparable to the local characteristic size, diffusion by the solid skeleton must be taken into account; and if the wavelengths are still reduced, all macroscopic propagating description becomes impossible. Here, we will consider only large wavelength situations where the diffusion theory can be ignored. Clearly, this is an attempt to directly model the geometry of the porous structure by defining a periodic unit cell that captures the intricate details of the porous material. As a result, this models an infinitely large matrix as if the porous structure within the unit cell were replicated in all other unit cells, without discontinuities in the porous structure between one unit cell and the adjacent one. Container effects are ignored, or at least negligible if the condition $L>a$ is well respected. At a given frequency, two equivalent relations linking the gradient of the macroscopic pressure to the macroscopic velocity are

$$
\rho_{0} \alpha_{i j}(\omega) \frac{\partial\langle\mathbf{v}\rangle_{j}}{\partial t}(\mathbf{r})=-\nabla_{i}\langle p\rangle(\mathbf{r})
$$

where $\rho_{0}$ is the equilibrium density of the fluid, and

$$
\phi\langle\mathbf{v}\rangle_{i}(\mathbf{r})=-\frac{1}{\eta} k_{i j}(\omega) \nabla_{j}\langle p\rangle(\mathbf{r}),
$$

where $\phi$ is the porosity and $\eta$ the viscosity of the fluid. Two second-order tensors, the dynamic tortuosity $\alpha_{i j}(\omega)$ and the dynamic permeability $k_{i j}(\omega)$, are defined by Eqs. (3) and (4). They only depend on frequency and on the geometry of the porous structure.

These two quantities, previously defined and studied by Johnson et al., ${ }^{6}$ not only characterize the response of the fluid to a spatially constant oscillating pressure gradient but also are relevant ingredients of the long-wavelength sound propagation theory. Indeed, for longwavelength sound propagation in the material, the dynamic tortuosity tensor plays a role similar to the electric permittivity tensor. 
When frequency decreases, the oscillatory flow locally becomes very similar to the static flow and Eq. (4) becomes

$$
\phi\langle\mathbf{v}\rangle_{i}=-\frac{1}{\eta} k_{0 i j} \nabla_{j}\langle p\rangle .
$$

This relation is the Darcy law, ${ }^{7}$ and the components $k_{0 i j}$ define the static viscous permeability tensor. When $\omega$ becomes very large, the effect of viscosity becomes negligible and $\alpha_{i j}(\omega)$ tends to the tortuosity tensor $\alpha_{\infty i j}$. The symmetry of $k_{0 i j}$ was demonstrated earlier for periodic structures by Ene and Sanchez-Palencia. ${ }^{8}$ This was followed by a demonstration of the symmetry of the inverse Fourier transform of the dynamic permeability. ${ }^{9}$ Some other properties of the inverse permeability tensor are also shown by Auriault. ${ }^{10}$ Numerical determination of the dynamic permeability can be found for a simple periodic rib duct; ${ }^{11}$ however, in this case, the symmetry property is practically useless since only one pressure gradient direction may be chosen.

Because the symmetry tensor property is relatively counterintuitive for apparently anisotropic porous structures, mathematical explanations are given in Sec. 2, and a straightforward consequence of this property for porous structures with trigonal and hexagonal symmetry is pointed out: that is, the invariance of the dynamic permeability tensor components in directions lying in the plane perpendicular to the axis of symmetry. Note that a similar property also exists for the permittivity tensor in uniaxial crystals. ${ }^{3}$ In Sec. 3, steady and unsteady flow simulations in a porous structure with hexagonal symmetry are reported, with detailed systematic evaluations of the tortuosity, static, and dynamic permeability tensor components in the plane perpendicular to the axis of symmetry.

\section{Symmetry of the dynamic viscous permeability tensor}

As discussed in Ref. 5, describing the periodic oscillating flow created in a porous medium by an external unit harmonic pressure gradient $\mathbf{e} e^{-i \omega t}$, and using three individual unit solicitation vectors $\mathbf{e}^{i}$ in three perpendicular directions, with components $\mathbf{e}_{j}^{i}=\delta_{i j}$, one has to solve in the fluid volume $\Omega_{f}$ the following set of scaled equations (unsteady scaled Stokes problem):

$$
\frac{-i \omega}{\nu} \mathbf{w}_{i}^{j}=-\boldsymbol{\nabla}_{i} \pi^{j}+\Delta \mathbf{w}_{j}^{j}+\delta_{i j} \quad \text { in } \Omega_{f}, \quad \boldsymbol{\nabla} \cdot \mathbf{w}^{j}=0 \quad \text { in } \Omega_{f}, \quad \mathbf{w}^{j}=0 \quad \text { on } \partial \Omega,
$$

where $\nu=\eta / \rho_{0}$. The solution to the problem described by Eq. (6) is fixed by adding the condition that $\pi^{j}$ is a spatially stationary or periodic field. This problem is relevant to sound propagation as long as the wavelength is large enough for the saturating fluid to behave as an incompressible fluid in volumes of the order of the homogenization volume (a period in the case of periodic structure).

From Eq. (4) and solution to Eq. (6), the viscous permeability components may be written in the form ${ }^{12}$

$$
k_{i j}(\omega)=\phi\left\langle\mathbf{w}_{i}^{j}\right\rangle=\phi\left\langle\mathbf{w}_{l}^{j} \delta_{i l}\right\rangle=\phi\left\langle\mathbf{w}_{l}^{j} \mathbf{e}_{l}^{i}\right\rangle=\phi\left\langle\mathbf{w}^{j} \cdot \mathbf{e}^{i}\right\rangle,
$$

where, from the left-hand side of the first equation in Eq. (6), $\left\langle\mathbf{w}^{j} \cdot \mathbf{e}^{i}\right\rangle$ is given by

$$
\left\langle\mathbf{w}^{j} \cdot \mathbf{e}^{i}\right\rangle=\frac{-i \omega}{\nu}\left\langle\mathbf{w}^{j} \cdot \mathbf{w}^{i}\right\rangle+\left\langle\mathbf{w}^{j} \cdot \boldsymbol{\nabla} \pi^{i}\right\rangle-\left\langle\mathbf{w}^{j} \cdot \Delta \mathbf{w}^{i}\right\rangle .
$$

The second term on the right-hand side of Eq. (8) is equal to 0. This can be shown in the following manner by rewriting

$$
\left\langle\mathbf{w}^{j} \cdot \boldsymbol{\nabla} \pi^{i}\right\rangle=\left\langle\boldsymbol{\nabla} \cdot\left(\mathbf{w}^{j} \pi^{i}\right)\right\rangle-\left\langle\pi^{i} \boldsymbol{\nabla} \cdot \mathbf{w}^{j}\right\rangle .
$$

First, from the local incompressibility condition [the second equation in Eq. (6)], the last term in Eq. (9) is strictly equal to zero. Second, using the spatial average theorem, Eq. (2), the first term on the right-hand side in Eq. (9) is rewritten as 


$$
\left\langle\boldsymbol{\nabla} \cdot\left(\mathbf{w}^{j} \pi^{i}\right)\right\rangle=\boldsymbol{\nabla} \cdot\left\langle\mathbf{w}^{j} \pi^{i}\right\rangle+\int_{\mathbf{x} \in \partial \Omega} f(\mathbf{x}-\mathbf{r}) \mathbf{n}(\mathbf{x}) \cdot \mathbf{w}^{j} \pi^{i} d \mathbf{x}
$$

Since the fields are spatially stationary or periodic, the first term on the right-hand side in Eq. (10) is equal to zero, and from the no-slip condition [the third equation in Eq. (6)], the second term is also equal to 0 . This proves that $\left\langle\mathbf{w}^{j} \cdot \nabla \pi^{i}\right\rangle=0$. To further simplify Eq. (8), the third term on the right-hand side can be rewritten

$$
\left\langle\mathbf{w}^{j} \cdot \boldsymbol{\Delta} \mathbf{w}^{i}\right\rangle=\left\langle\frac{\partial}{\partial x_{m}}\left(\mathbf{w}^{j} \cdot \frac{\partial}{\partial x_{m}} \mathbf{w}^{i}\right)-\left(\frac{\partial}{\partial x_{m}} \mathbf{w}^{j}\right) \cdot \frac{\partial}{\partial x_{m}} \mathbf{w}^{i}\right\rangle .
$$

It is shown by using the spatial average theorem that the first term on the right-hand side is equal to 0 . Finally, from these simplifications, $k_{i j}(\omega)$ can be written in a symmetrical form in $i$ and $j$,

$$
k_{i j}(\omega)=-\frac{i \omega \phi}{\nu}\left\langle\mathbf{w}^{j} \cdot \mathbf{w}^{i}\right\rangle+\phi\left\langle\left(\frac{\partial}{\partial x_{m}} \mathbf{w}^{j}\right) \cdot \frac{\partial}{\partial x_{m}} \mathbf{w}^{i}\right\rangle .
$$

It has been observed by Brown ${ }^{13}$ that the same type of equation also describes the electrical current density in a conducting fluid saturating a nonconducting porous frame. Consequently, as for the permeability tensor, one can identify the tortuosity tensor elements from the resistivity tensor elements of the saturated medium, normalized by the resistivity of the saturating fluid, and multiplied by porosity.

These demonstrations clearly show that the viscous permeability tensor and tortuosity tensor are symmetric, $k_{j i}(\omega)=k_{i j}(\omega)$ and $\alpha_{j i}(\omega)=\alpha_{i j}(\omega)$. As a consequence, there exists a system of orthogonal axes, the principal axes, where the tensors have only diagonal elements different from zero (see, for example, Refs. 1-3). If the medium presents trigonal, tetragonal, or hexagonal symmetry, the axis of symmetry $Z$ must coincide with one of the principal axes. Moreover, the invariance of the system through some discrete rotations along this axis necessarily means that the two transverse eigenvalues of the tensors are the same (transverse isotropy). In particular, the static permeability $k_{0 i j}$, the tortuosity $\alpha_{\infty i j}$, and the dynamic permeability $k_{i j}(\omega)$ have values $k_{0 Z}, \alpha_{\infty Z}$, and $k_{Z}(\omega)$ along axis $Z$, and different unique values $k_{0 P}, \alpha_{\infty P}$, and $k_{P}(\omega)$ for all directions orthogonal to $Z$.

Parameters $\alpha_{\infty}$, and $k_{0}$, and $k(\omega)$ are important for the prediction of the acoustic properties of porous media and many numerical simulations have been performed to predict these parameters in periodic structures (see Ref. 12, and references therein). The constraint which exists when the structure has the uniaxial symmetry can provide a test for the precision of the simulations. An example is given in Sec. 3 , where the tortuosity $\alpha_{\infty P}$, the static permeability $k_{0 P}$, and the dynamic permeability $k_{P}(\omega)$ are evaluated in a hexagonal porous structure.

\section{Flow simulation in a hexagonal porous structure}

At $\omega=0$, Eq. (6), with the condition that $\pi^{j}$ is a stationary field, simply describe the viscous fluid motion in steady state regime. This is the steady Stokes problem for periodic structures, where $\mathbf{w}_{0}$ is the scaled static velocity field in the pore in square meters. Thus, the components $k_{0 i j}$ defining the static viscous permeability tensor are simply given by

$$
k_{0 i j}=\phi\left\langle\mathbf{w}_{0}^{j} \cdot \mathbf{e}^{i}\right\rangle .
$$

At the opposite frequency range, when $\omega$ becomes very large, the viscous boundary layer becomes negligible and the fluid tends to behave as a perfect one, having no viscosity. Under these conditions, the perfect incompressible fluid formally behaves according to the electric problem. ${ }^{6,13}$ The components $\alpha_{\infty i j}$ defining the tortuosity are now given by ${ }^{5}$ 


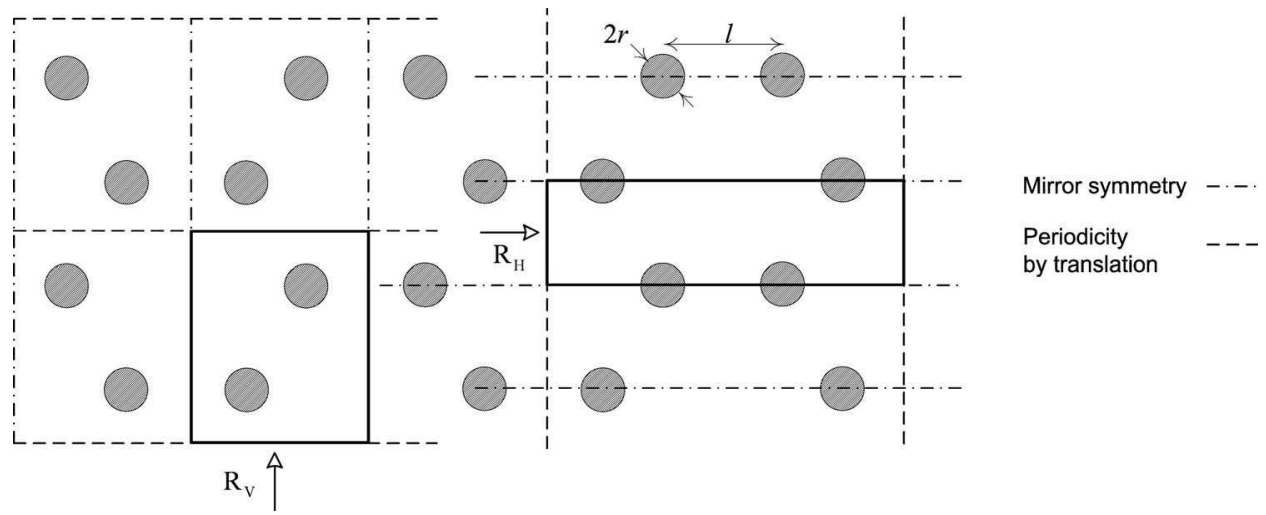

Fig. 1. Identification of two-dimensional rectangular periodic unit cells for vertical $\left(R_{V}\right)$ and horizontal $\left(R_{H}\right)$ wave propagations through a hexagonal lattice of solid cylinders.

$$
\alpha_{\infty i j}^{-1}=\left\langle\mathbf{E}^{j} \cdot \mathbf{e}^{i}\right\rangle,
$$

where $\alpha_{\infty i j}^{-1}$ denotes the inverse of the tortuosity tensor $\alpha_{\infty i j}$, and $\mathbf{E}$ is the scaled electric field that solves the corresponding electrical conduction problem for a porous medium filled with a conducting fluid and having an insulating solid phase, i.e.,

$$
\mathbf{E}=-\nabla \varphi+\mathbf{e} \quad \text { in } \Omega_{f}, \quad \nabla \cdot \mathbf{E}=0 \quad \text { in } \Omega_{f}, \quad \mathbf{E} \cdot \mathbf{n}=0 \quad \text { on } \partial \Omega,
$$

and $\varphi$ is a spatially stationary or periodic scalar field representing the deviatoric part of the electric potential.

Taking the external macroscopic field $\mathbf{e}$ in the horizontal and vertical directions, numerical computations of the fields $\mathbf{E}, \mathbf{w}_{0}$, and $\mathbf{w}$ were performed on the periodic rectangles $R_{H}$ and $R_{V}$ of the hexagonal porous structure depicted in Fig. 1, where $l$ is the length between two cylinders and $r$ is the radius of a solid cylinder.

The radius of the cylinders is $r=(1-\phi) \Lambda^{\prime} / \phi \approx 0.163 \mathrm{~mm}$, and $l$ $=\sqrt{4 \pi r\left(\Lambda^{\prime}+r\right) /(3 \sqrt{3})} \approx 0.894 \mathrm{~mm}$; corresponding to a porosity $\phi=0.92$ and a thermal characteristic length $\Lambda^{\prime}=1.87 \mathrm{~mm}$ (which is equal to twice the interconnected pore fluid volume to pore wet surface ratio). The corresponding scaled electric, steady, and unsteady Stokes problems were solved using a finite-element commercial code. ${ }^{14}$ No-slip boundary conditions at the pore walls, and periodicity of $\pi_{0}, \mathbf{w}_{0}$, and $\mathbf{w}$ were prescribed. Neumann boundary conditions on the fluid-solid interface, and periodicity on the inlet-outlet surfaces were used for $\varphi$. Additional Neumann boundary conditions are set in the remaining lateral borders due to the symmetries of the problems.

The number of elements and their distribution in the fluid phase regions of $R_{H}$ and $R_{V}$ were varied, with attention paid especially to the throat and the near-wall areas, to examine the accuracy and convergence of the field solutions. As an illustration, it can be seen in Fig. 2 that once convergence is achieved, there is an almost perfect superposition of the diagonal terms of the dynamic permeability tensor values [i.e., $k_{11}(\omega)$ and $k_{22}(\omega)$ ] obtained for the external orthogonal solicitations. Furthermore, we show in Figs. 3 and 4 the two components of the static and high frequency scaled patterns obtained for excitations along the two principal directions in the plane perpendicular to the axis of symmetry of the periodic geometry. As previously noticed by several authors, such as Martys and Garboczi ${ }^{15}$ due to the nonslip condition, the fluid flow paths are more concentrated than the electric-current paths arising when effects of fluid viscosity are minimal. Once the flow fields are known, the static and dynamic viscous permeability and the tortuosity tensors are computed using Eqs. (13), (7), and (14), respectively. This yields the following tensors in the asymptotic regimes: 


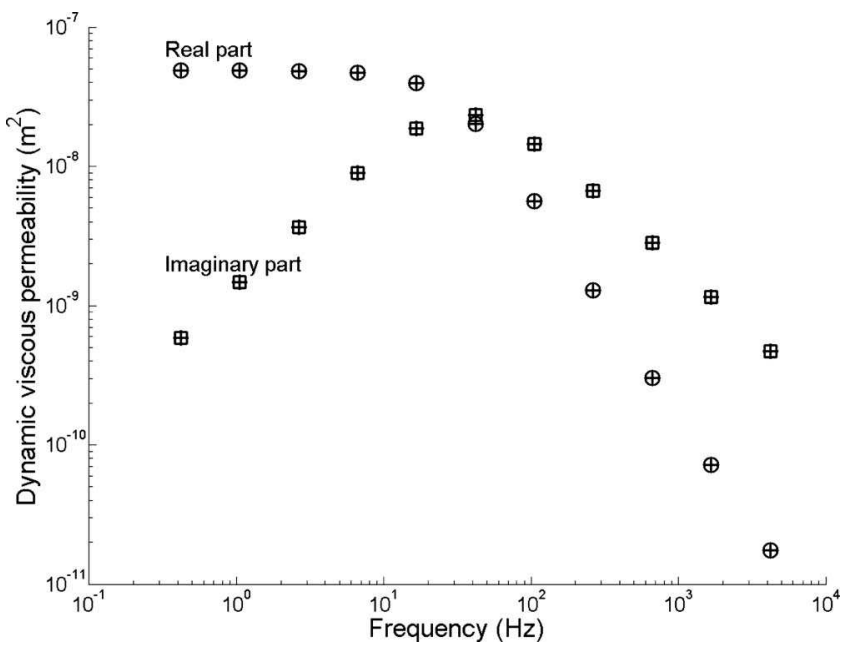

Fig. 2. Dynamic viscous permeability of a hexagonal lattice of solid cylinders: There is an almost perfect superposition of the permeability values computed from any orthogonal line pairs perpendicular to the sixfold axis of symmetry, suggesting that this property might be used for a convergence check.
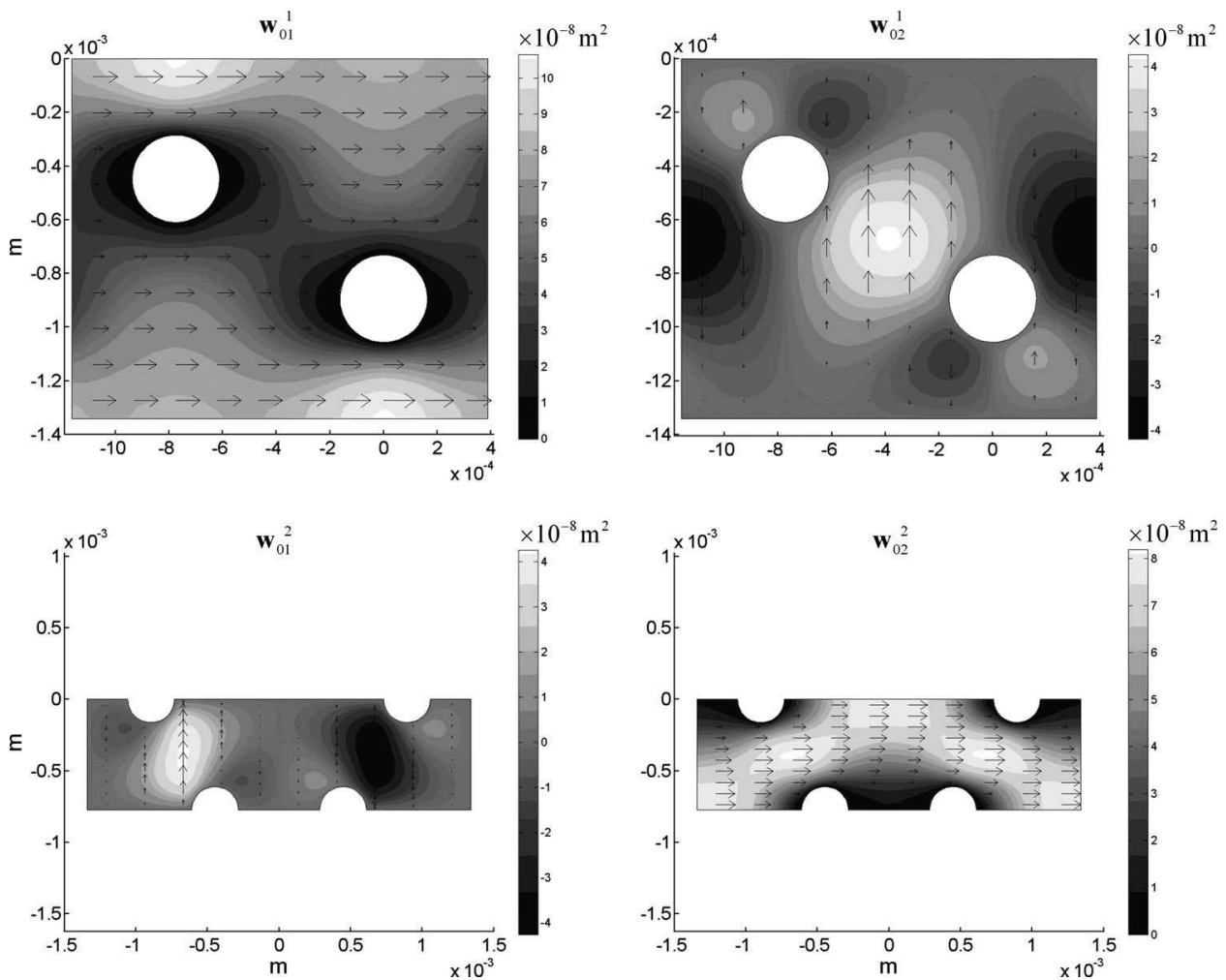

Fig. 3. Vertical (top) and horizontal (bottom) static scaled velocity fields obtained by solving the steady Stokes problem in principal directions of the periodic porous structure. 

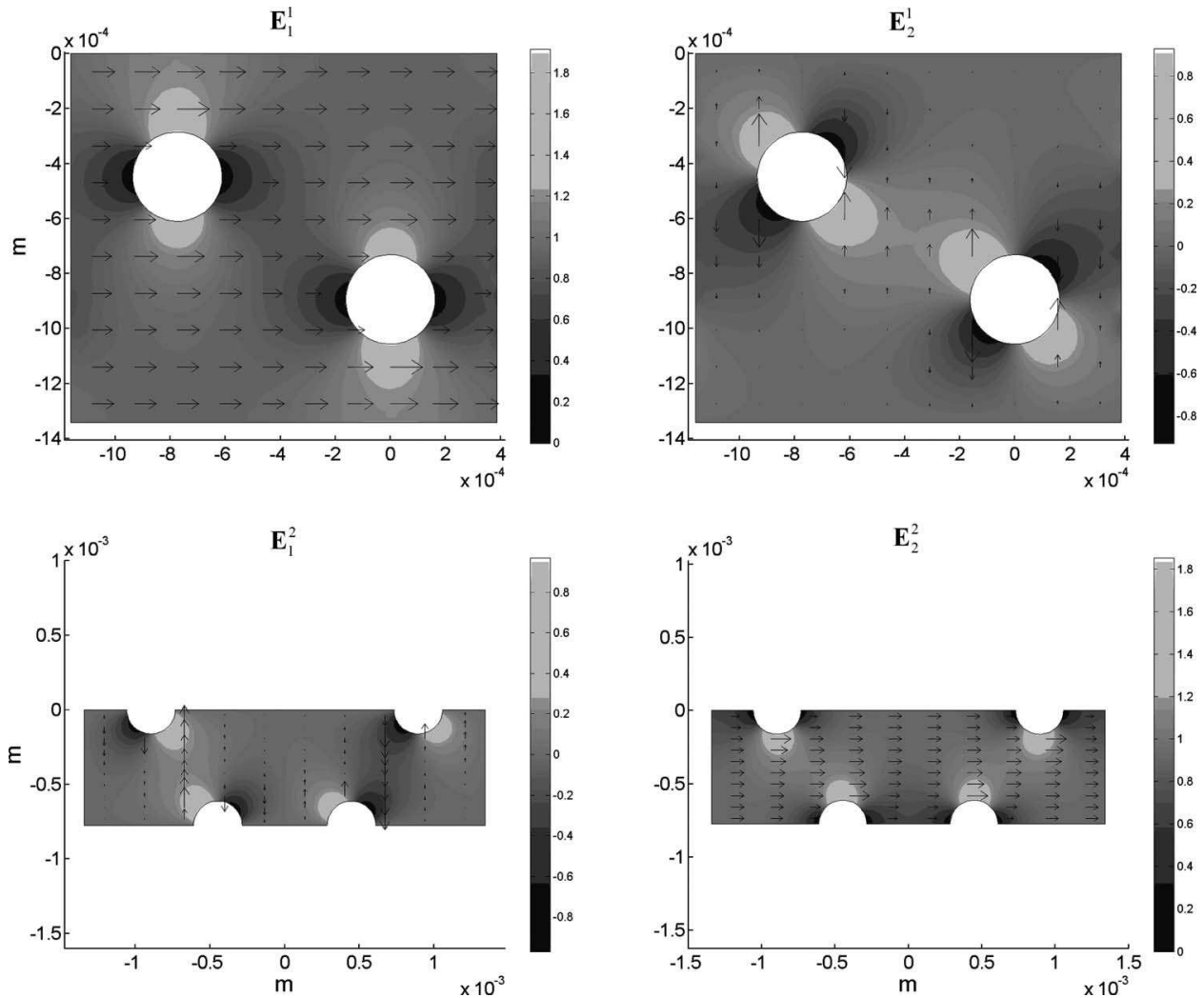

Fig. 4. Vertical (top) and horizontal (bottom) scaled electric fields obtained by solving the electric problem in principal directions of the periodic porous structure.

$$
\left[\begin{array}{ll}
k_{011} & k_{012} \\
k_{021} & k_{022}
\end{array}\right]=\varphi\left[\begin{array}{ll}
\left\langle\mathbf{w}_{01}^{1}\right\rangle & \left\langle\mathbf{w}_{01}^{2}\right\rangle \\
\left\langle\mathbf{w}_{02}^{1}\right\rangle & \left\langle\mathbf{w}_{02}^{2}\right\rangle
\end{array}\right]=\left[\begin{array}{cc}
4.9420 & 0 \\
0 & 4.9353
\end{array}\right] \times 10^{-8} \mathrm{~m}^{2},
$$

and

$$
\left[\begin{array}{cc}
\alpha_{\infty 11} & \alpha_{\infty 12} \\
\alpha_{\infty 21} & \alpha_{\infty 22}
\end{array}\right]=\frac{1}{\left\langle\mathbf{E}_{1}^{1}\right\rangle\left\langle\mathbf{E}_{2}^{2}\right\rangle-\left\langle\mathbf{E}_{2}^{1}\right\rangle\left\langle\mathbf{E}_{1}^{2}\right\rangle} \cdot\left[\begin{array}{cc}
\left\langle\mathbf{E}_{2}^{2}\right\rangle & -\left\langle\mathbf{E}_{2}^{1}\right\rangle \\
-\left\langle\mathbf{E}_{1}^{2}\right\rangle & \left\langle\mathbf{E}_{1}^{1}\right\rangle
\end{array}\right]=\left[\begin{array}{cc}
1.0802 & 0 \\
0 & 1.0801
\end{array}\right] .
$$

In both cases, the relative differences between the horizontal and vertical directions are less than $0.13 \%$. Also, the nondiagonal terms are numerically equal to zero. These results are consistent with theoretical results presented in Sec. 2, and, as a consequence, prove the validity of the numerical implementation.

Finally, these results provide, in the limit of the precision of the finite-element method, a numerical illustration of the symmetry property of the dynamic viscous permeability tensor. In a plane perpendicular to the axis of a porous material with hexagonal symmetry, tortuosity, static, and dynamic viscous permeability tensors are reduced to a constant diagonal element.

\section{Conclusion}

A simple illustration of a somewhat nonintuitive property of the dynamic viscous permeability tensor in rigid-frame porous media was presented in the case of a wavelength much larger than the pore size. For periodic porous structures with hexagonal symmetry presenting different 
local configurations to a wave propagating in different directions in the plane perpendicular to the axis of symmetry, the dynamic permeability tensor is diagonal and constant in the different directions. This property can notably be used for error estimation in numerical computations.

\section{References and links}

${ }^{1}$ P. M. Adler, "Flow in spatially periodic structures," in Porous Media: Geometry and Transports, edited by H. Brenner et al. (Butterworths, London, 1992), pp. 144-185.

${ }^{2}$ C. Y. Wang, "Stokes slip flow through square and triangular arrays of circular cylinders," Fluid Dyn. Res. 32, 233-246 (2003).

${ }^{3}$ M. Born and E. Wolf, "Optics of crystals," in Principles of Optics (Cambridge University Press, Cambridge, 2002), pp. 790-849.

${ }^{4}$ S. Torquato, "Relationship between permeability and diffusion-controlled trapping constant of porous media," Phys. Rev. Lett. 64, 2644-2646 (1990).

${ }^{5}$ D. Lafarge, "Modèles linéaires de propagation," in Milieux poreux et poreux stratifiés (Linear Models of Propagation in Porous Media and Stratified Porous), Matériaux et Acoustique (Materials and Acoustics), edited by M. Bruneau and C. Potel (Lavoisier, Paris, 2006), pp. 143-187.

${ }^{6}$ D. L. Johnson, J. Koplik, and R. Dashen, "Theory of dynamic permeability and tortuosity in fluid-saturated porous media,” J. Fluid Mech. 176, 379-402 (1987).

${ }^{7}$ H. Darcy, Les Fontaines Publiques de la Ville de Dijon (Victor Dalmont, Paris, 1856).

${ }^{8}$ H. I. Ene and E. Sanchez-Palencia, "Équations et phénomènes de surface pour l'écoulement dans un modéle de milieu poreux (Equations and surface phenomena for flow through a porous media model), J. Mec. 14, 73-108 (1975).

${ }^{9}$ E. Sanchez-Alencia, "Fluid flow in porous media," in Non-Homogeneous Media and Vibration Theory (Springer, Berlin, 1980), pp. 149-154.

${ }^{10}$ J.-L. Auriault, "Dynamic behaviour of a porous medium saturated by a Newtonian fluid,” Int. J. Eng. Sci. 18, 775-785 (1980).

${ }^{11}$ J.-L. Auriault, L. Borne, and R. Chambon, "Dynamics of porous saturated media, checking of the generalized law of Darcy," J. Acoust. Soc. Am. 77, 1641-1650 (1985).

${ }^{12}$ C. Perrot, F. Chevillotte, and R. Panneton, "Dynamic viscous permeability of an open-cell aluminium foam: Computations vs experiments," J. Appl. Phys. 103, 024909-8 (2008)

${ }^{13}$ R. J. S. Brown, "Connection between formation factor for electrical-resistivity and fluid-solid coupling factor in Biot equations for acoustic waves in fluid-filled porous media," Geophysics 45, 1269-1275 (1980).

${ }^{14}$ Comsol 3.4, WTC-5 pl. Robert Schuman, 38000 Grenoble, France.

${ }^{15} \mathrm{~N}$. Martys and E. J. Garboczi, "Length scales relating the fluid permeability and electrical conductivity in random two-dimensional model porous media," Phys. Rev. B 46, 6080-6090 (1992). 